\title{
Диэлектрическая спектроскопия и особенности механизма фазового перехода полупроводник-металл в пленках $\mathrm{VO}_{2}$
}

\author{
(C) А.В. Ильинский ${ }^{1}$, Р.А. Кастро ${ }^{2}$, М.Э. Пашкевич ${ }^{3}$, Е.Б. Шадрин ${ }^{1}$ \\ ${ }^{1}$ Физико-технический институт им. А.Ф. Иоффе Российской академии наук, \\ 194021 Санкт Петербург, Россия \\ ${ }^{2}$ Российский государственный педагогический университет им. А.И. Герцена, \\ 191186 Санкт-Петербург, Россия \\ ${ }^{3}$ Санкт-Петербургский политехнический университет Петра Великого, \\ 195251 Санкт-Петербург, Россия \\ E-mail: shadr.solid@mail.ioffe.ru
}

Поступила в Редакцию 25 сентября 2019 г.

В окончательной редакции 30 сентября 2019 г.

Принята к публикации 30 сентября 2019 г.

В интервале $0.1-10^{6}$ Гц изучена температурная трансформация частотных зависимостей тангенса угла диэлектрических потерь $\operatorname{tg} \delta(f)$, а также диаграммы Коула-Коула для нелегированных пленок диоксида ванадия. Измерения проведены в температурном интервале $T=273-373 \mathrm{~K}$. Показано, что вид диаграмм Коула-Коула для всех пленок слабо зависит от температуры в указанном интервале, тогда как частоты $f_{0}$, соответствующие максимумам функции $\operatorname{tg} \delta(f)$, увеличиваются с ростом температуры. Измерены петли температурного гистерезиса частотных положений максимумов $f_{0}(T)$. При интерпретации данных диэлектрической спектроскопии использована комплексная эквивалентная электрическая схема образца, позволившая выявить в специально не легированных пленках $\mathrm{VO}_{2}$ наличие двух типов зерен, различающихся электрическими свойствами. Присутствие двух типов зерен определяет особенности механизма фазового перехода полупроводник-металл в пленках $\mathrm{VO}_{2}$.

Ключевые слова: диоксид ванадия $\mathrm{VO}_{2}$, пленки $\mathrm{VO}_{2}$, фазовый переход, корреляционные эффекты, диэлектрическая спектроскопия, атомно-силовая микроскопия.

DOI: 10.21883/FTP.2020.02.48910.9267

\section{1. Введение}

Диоксид ванадия $\left(\mathrm{VO}_{2}\right)$ претерпевает фазовый переход полупроводник-металл (ФППМ) при температуре $T_{c}=340 \mathrm{~K}[1]$. Ниже этой температуры материал имеет моноклинную симметрию кристаллической решетки и полупроводниковые свойства (с шириной запрещенной зоны $\sim 0.8$ эВ). Выше $T_{c}$ симметрия решетки меняется на тетрагональную, и диоксид ванадия приобретает металлическую проводимость. Благодаря присутствию электронных корреляций ФППМ в $\mathrm{VO}_{2}$ приобретает комплексную природу и оказывается состоящим из трех стадий: двух, имеющих место в системе сильно коррелированных электронов (переходы Мотта), и одной, сводящейся к трансформации симметрии кристаллической решетки (переход Пайерлса). При этом ФППМ сопровождается скачкообразным изменением проводимости и оптических свойств пленок $\mathrm{VO}_{2}$, определяя принцип действия целого ряда устройств (термореле, болометров, оптических лимитеров, оптических элементов памяти и др.) [2-4].

Совершенствование конструкции упомянутых устройств требует создания возможности управления температурой ФППМ с целью оптимизации энергетического порога их срабатывания. Этот порог определяется теплотой перехода и величиной зазора между рабочей температурой устройства и температурой ФППМ.
Последней можно управлять за счет изменения концентрации донорных или акцепторных примесей в нанокристаллитах $\mathrm{VO}_{2}$ пленки.

В связи с этим в настоящей работе мы сосредоточим свое внимание на таком малоизученном варианте управления $T_{c}$, как создание условий для инициирования процесса спонтанного возникновения донорных кислородных вакансий внутри нанокристаллитов $\mathrm{VO}_{2}$.

Для контроля за данным процессом мы используем методы диэлектрической спектроскопии (ДС), которые открывают новые возможности изучения электронных состояний пленочных кристаллических материалов и, в частности, пленок диоксида ванадия. Достоинством методики ДС является ее высокая чувствительность к изменению электрофизических параметров предельно тонких пленок нанокристаллических материалов [5].

\section{2. Образцы и методика эксперимента}

Пленки $\mathrm{VO}_{2}$ толщиной $1400 \AA$ были синтезированы на ситалловой подложке одним из вариантов метода взрывного испарения (сублимации), а именно методом Харриса и Зигеля [6], с использованием порошка $\mathrm{VO}_{2}$. При этом пакеты мелко раздробленного материала дискретными порциями подавались в испаритель, разогретый до температуры $770^{\circ} \mathrm{C}$. Взрывная сублимация 
порошка обеспечивала, независимо от упругости паров компонент, идентичность состава паров над испарителем и вблизи подложки. В качестве подложки была использована ситалловая пластина ( $\mathrm{SiAl}$ ) стандартных размеров $40 \times 60 \times 0.7$ мм. Температура подложки была ниже температуры испарителя и составляла $470^{\circ} \mathrm{C}$, что обеспечивало высокую поверхностную подвижности атомов при одновременной стабильности протекания процесса осаждения материала, что исключало опасность обратного испарения материала с подложки. Это позволяло формировать на ситалловой подложке однородную пленку постоянного состава $\mathrm{VO}_{2}$. Особое внимание уделялось поддержанию постоянства условий синтеза, что является принципиально важным для обеспечения вдоль поверхности пленки однородности стехиометрии по кислороду ввиду того, что локальное изменение концентрации кислорода способно привести к маскирующему исследуемые эффекты изменению температуры перехода ФППМ.

Морфология пленок $\mathrm{VO}_{2}$ контролировалась путем анализа изображений, полученных с помощью атомносилового микроскопа. Площадь поверхности пленочных образцов составляла $10 \times 10 \mathrm{Mм}^{2}$, толщина пленок $1100 \AA$, удельное сопротивление $-7 \cdot 10^{6}$ Ом $\cdot$ м при комнатной температуре.

Измерение диэлектрических характеристик и исследования их температурной зависимости выполнялись на диэлектрическом спектрометре марки „Alpha-Beta Impedance Analyzer" фирмы Novocontrol Technologies. Образец пленки диоксида ванадия, синтезированной на ситалловой подложке, помещался между плоскими электродами диаметром 20 мм. Измерялась амплитуда $I_{0}$ тока $I(t)$, протекавшего через образец перпендикулярно поверхности пленки при подаче эталонного синусоидального напряжения $U(t)$ амплитудой 1 В. Измерялась также разность фаз $\varphi$ колебаний $U(t)$ и $I(t)$. С помощью конвертера эти данные с применением разработанных фирмой Novocontrol Technologies компьютерных программ преобразовывались в данные о физических характеристиках образца $(\operatorname{tg} \delta$ - такгенс угла диэлектрических потерь, $\varepsilon^{\prime}, \varepsilon^{\prime \prime}$ - действительная и мнимая части диэлектрической проницаемости). Геометрическая емкость пустой ячейки $-C_{0}$.

Измерения частотных зависимостей $\operatorname{tg} \delta(f), \varepsilon^{\prime}(f)$, $\varepsilon^{\prime \prime}(f)$ были выполнены в интервале от $10^{-1}$ Гц до 1 МГц, температура образца $T$ изменялась в процессе измерений от 273 до $373 \mathrm{~K}$.

\section{3. Результаты эксперимента}

На рис. 1 представлено атомно-силовое изображение специально не легированной пленки $\mathrm{VO}_{2}$, на вставке к рис. 1 - гистограмма распределения относительной доли зерен $\rho$ по размерам $L$. Обращает на себя внимание наличие двух типов зерен: „крупных“ и „мелких“. Здесь заметим также, что петли температурного гистерезиса

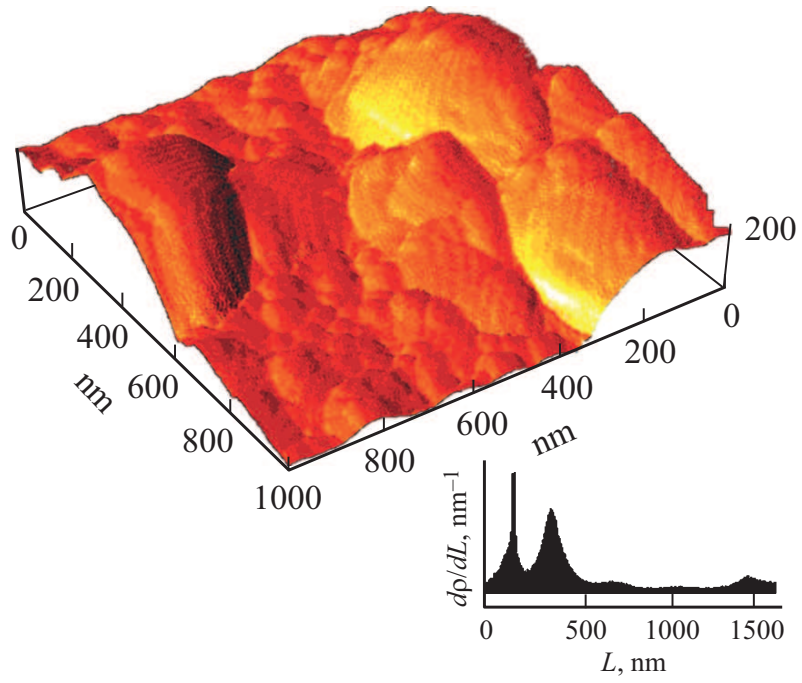

Рис. 1. Атомно-силовое изображение поверхности пленки диоксида ванадия на подложке из $\mathrm{Al}_{2} \mathrm{O}_{3}$. и гистограмма распределения зерен по размерам.

электропроводности пленки, измеренные по стандартной методике [7], обнаруживают наличие ступеньки на нагревной ветви петли гистерезиса.

На рис. 2 представлена частотная зависимость тангенса угла диэлектрических потерь $\operatorname{tg} \delta(f)$ и диаграмма Коула-Коула для нелегированной пленки $\mathrm{VO}_{2}$ при температуре $303 \mathrm{~K}$. Точки - результат эксперимента, сплошные линии - расчет (см. далее). Видно, что наряду с основным максимумом $\operatorname{tg} \delta\left(f_{0 \mathrm{I}}\right)$ на частоте $f_{0 \mathrm{I}}=48$ кГц, наблюдается второй максимум на существенно меньшей частоте, $\operatorname{tg} \delta\left(f_{0 \text { II }}\right)$ на частоте $f_{\text {0II }}=0.1$ Гц. Диаграмма Коула-Коула представляет собой большую высокочастотную полуокружность, к которой со стороны высоких значений действительной части диэлектрической проницаемости (справа, что соответствует низким частотам) примыкает меньшая по диаметру полуокружность. Наличие второго (неосновного) низкочастотного максимума $\operatorname{tg} \delta(f)$ и связанной с ним второй (низкочастотной) полуокружности - новый результат, на котором мы сосредоточили свое внимание в настоящей статье.

На рис. 3 представлены экспериментальные частотные зависимости тангенса угла диэлектрических потерь и диаграммы Коула-Коула для пленки $\mathrm{VO}_{2}$ при различных температурах в интервале 293-343 К, полученные с шагом $5 \mathrm{~K}$. С повышением температуры оба максимума $\operatorname{tg} \delta(f)$ смещаются в сторону высоких частот. Высокочастотный максимум при изменении температуры в диапазоне 293-323 K смещается на меньший частотный интервал, чем при изменении температуры в диапазоне $323-343$ К. Низкочастотный максимум, напротив, смещается в диапазоне $293-323 \mathrm{~K}$ на больший частотный интервал, чем в диапазоне $323-343 \mathrm{~K}$, в котором положение этого максимума практически 

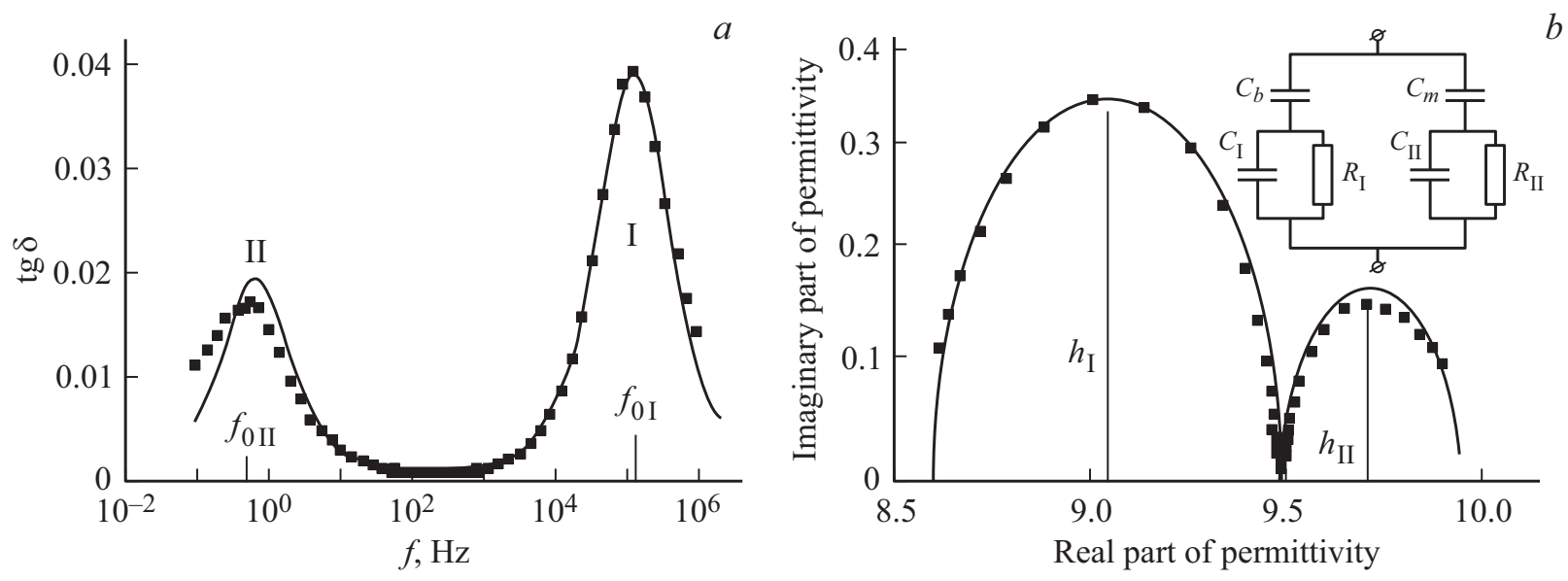

Рис. 2. Частотные зависимости тангенса угла диэлектрических потерь $\operatorname{tg} \delta(f)(a)$ и диаграмма Коула-Коула для нелегированной пленки $\mathrm{VO}_{2}$ при температуре $T=303 \mathrm{~K}$. $b$ : на вставке - эквивалентная электрическая схема. Точки - результат эксперимента, сплошные линии - результат расчета $\left(C_{b}=6.2 \Pi \Phi, C_{\mathrm{I}}=33 \Pi \Phi, R_{\mathrm{I}}=56.8\right.$ кОм, $C_{m}=7.5 \Pi \Phi, C_{\mathrm{II}}=127 \Pi \Phi, R_{\mathrm{II}}=2.8 \Gamma$ Ом, $C_{0}=1.4 \Pi \Phi$.)
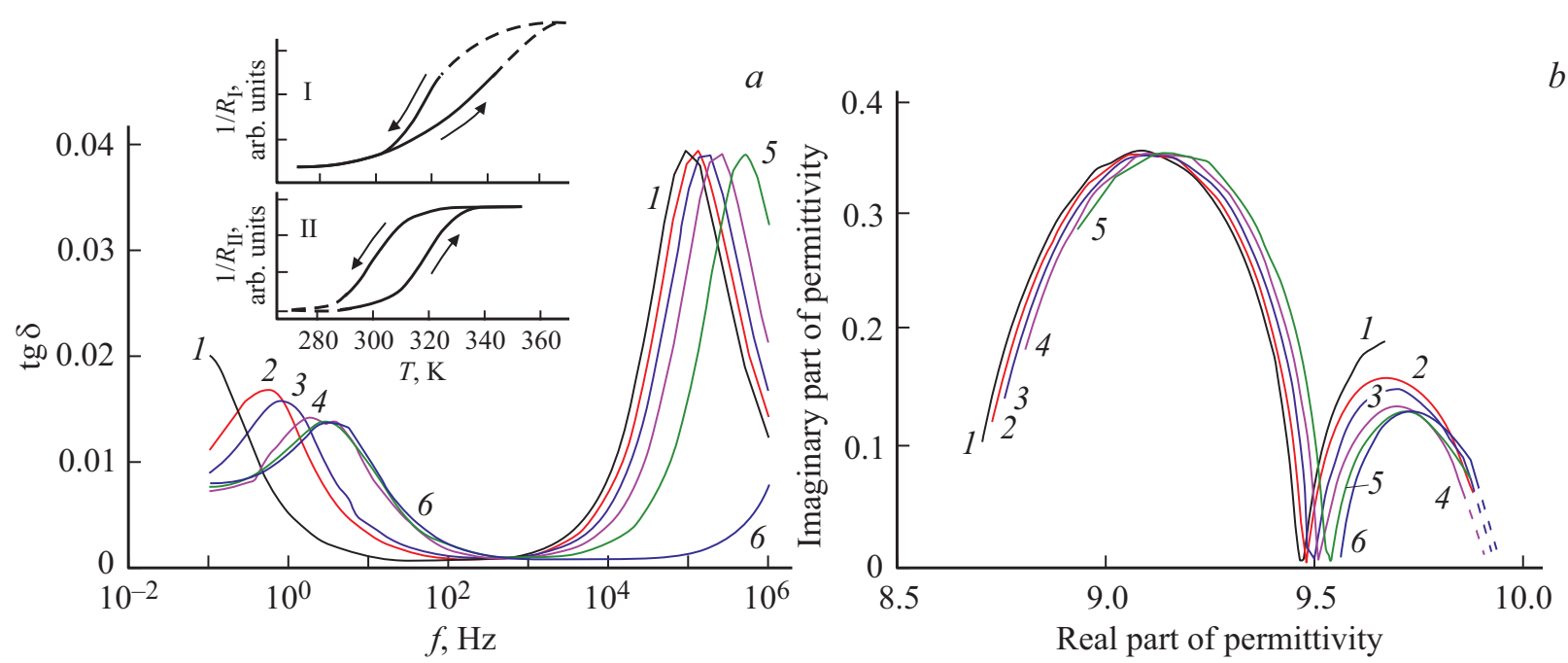

Рис. 3. Частотные зависимости тангенса угла диэлектрических потерь $\operatorname{tg} \delta(f)$ и диаграммы Коула-Коула для нелегированной пленки $\mathrm{VO}_{2}$ (эксперимент) при $T, \mathrm{~K}: 1-293,2-303,3-313,4-323,5-333,6-343$. На вставке $(a)-$ температурные гистерезисы проводимостей $1 / R_{\mathrm{I}}$ и $1 / R_{\mathrm{II}}$.

неизменно. На вставке к рис. 3 представлены петли температурного гистерезиса частотного положения максимумов $\operatorname{tg} \delta(f)$, а именно, максимумов $f_{\text {оI }}(T)$ и $f_{\text {0II }}(T)$, положения которых, как указано далее, пропорциональны проводимостям совокупностей зерен, соответствующих максимумам I и II. Середина нагревной ветви петли температурного гистерезиса максимума I, стандартно принимаемая за температуру фазового перехода, соответствует температуре структурного фазового перехода Пайерлса $T_{c \mathrm{I}}=340 \mathrm{~K}$, тогда как петля гистерезиса для максимума II сильно смещена в сторону низких температур, и для нее оказывается, что $T_{c \mathrm{II}}=320 \mathrm{~K}$.

Отметим, что, в противоположность $\operatorname{tg} \delta(f)$, вид диаграммы Коула-Коула слабо меняется при изменении температуры: большая (высокочастотная) полуокружность при своем неизменном радиусе $h_{\mathrm{I}}$ смещается вправо, тогда как радиус малой (низкочастотной) полуокружности $h_{\text {II }}$ уменьшается с температурой, причем ее правая крайняя точка остается неподвижной.

Наличие двух максимумов функции $\operatorname{tg} \delta(f)$ и двух полуокружностей на диаграмме Коула-Коула (рис. 2), несовпадение $T_{c}$ для двух петель температурного гистерезиса численных значений частот $f_{0}$, а также наличие ступеньки на главной петле температурного гистерезиса электропроводности пленки (вставка к рис. 3) представляют собой широкий набор экспериментальных данных. Это позволяет нам перейти к разработке комплексной эквивалентной схемы электрических параметров пленки $\mathrm{VO}_{2}$. 


\section{4. Обработка результатов измерений на базе электрических эквивалентных схем}

В нашей предыдущей работе [8] при обработке экспериментальных данных по частотной зависимости $\operatorname{tg} \delta(f)$ нелегированной пленки $\mathrm{VO}_{2}$ нами предполагалось, что эквивалентной схемой образца является параллельное соединение сопротивления $R$ и электроемкости $C_{\mathrm{I}}$ c включением последовательно с данной парой элементов дополнительной емкости $C_{b}$. Здесь $1 / R$ олицетворяет электрическую проводимость пленки, $C_{\mathrm{I}}$ - электрическую емкость пленки, $C_{b}$ соответствует электрической емкости подложки с бесконечно большим омическим сопротивлением. Расчеты частотных зависимостей, выполненные стандартным символическим методом на основе такой одноконтурной эквивалентной схемы, позволяют получить [8] частотную зависимость $\operatorname{tg} \delta(\omega)$, где $\omega=1 \pi f$, а также полуокружность на диаграмме Коула-Коула:

$$
\begin{aligned}
\operatorname{tg} \delta & =\left(R \omega C_{b}\right) /\left[1+R^{2} \omega^{2} C_{\mathrm{I}}\left(C_{\mathrm{I}}+C_{b}\right)\right], \\
\varepsilon^{\prime \prime}= & \left\{\varepsilon^{\prime} C_{b}\left(2 C_{\mathrm{I}}+C_{b}\right) /\left[C_{0}\left(C_{\mathrm{I}}+C_{b}\right)\right]-\left(\varepsilon^{\prime}\right)^{2}\right. \\
& \left.-C_{\mathrm{I}} C_{b}^{2} /\left[C_{0}^{2}\left(C_{\mathrm{I}}+C_{b}\right)\right]\right\}^{1 / 2} .
\end{aligned}
$$

Приравнивая к нулю производную по частоте функции $\operatorname{tg} \delta(f)$, получим, что положение максимума на шкале частот $f_{0}$ прямо пропорционально проводимости пленки $1 / R$. Анализ указанной схемы показывает, что с уменьшением $R$ максимум $\operatorname{tg} \delta(f)$ сдвигается в сторону высоких частот практически без изменения численного значения его ординаты в точке максимума.

Заметим здесь, что корнями уравнения (2) являются значения $\varepsilon^{\prime}=C_{b} / C_{0}$ (соответствует пределу при $\omega \rightarrow 0-$ правый по оси абсцисс корень функции $\varepsilon^{\prime \prime}\left(\varepsilon^{\prime}\right)$ на диаграмме Коула-Коула, см. рис. 1) и $\varepsilon^{\prime}=\left(C_{\mathrm{I}} C_{b}\right) /\left[\left(C_{\mathrm{I}}+C_{b}\right) C_{0}\right] \quad$ (соответствует пределу при $\omega \rightarrow \infty-$ левый корень функции $\varepsilon^{\prime \prime}\left(\varepsilon^{\prime}\right)$ на диаграмме Коула-Коула). Радиус полуокружности равен полуразности корней, т. е. $h=C_{b}^{2} /\left[2\left(C_{\mathrm{I}}+C_{b}\right) C_{0}\right]$. Аналитическое выражение (2) для функции $\varepsilon^{\prime \prime}\left(\varepsilon^{\prime}\right)$ показывает также, что параметры диаграммы Коула-Коула зависят только от емкостей $C_{\text {I }}$ и $C_{b}$, но не зависят от $R$.

Для нелегированной пленки $\mathrm{VO}_{2}$ новые эксперименты, однако, показывают, что наблюдаются два максимума на частотной зависимости $\operatorname{tg} \delta(f)$ и имеет место более сложная структура диаграммы Коула-Коула в виде двух полуокружностей (рис. 2). Для интерпретации этих результатов мы вынуждены использовать более сложную двухконтурную схему (вставка к рис. 2). Выбор двухконтурной схемы - первый этап нашего алгоритма обработки результатов измерений.

Как и для случая одноконтурной схемы, символическим методом нами получено аналитические выражения частотной зависимости $\operatorname{tg} \delta(f)$ и частотных зависимостей действительной, $\varepsilon^{\prime}(\omega)$, и мнимой, $\varepsilon^{\prime \prime}(\omega)$, частей диэлектрической проницаемости пленочного образца для усложненной эквивалентной схемы:

$$
\begin{aligned}
& \operatorname{tg} \delta(f)=-(D W+F V) /(F W-D V), \\
\mathcal{\varepsilon}^{\prime}(\omega)= & (F W-D V)\left(W^{2}+V^{2}\right) / \omega C_{0}\left[(D W+F V)^{2}\right. \\
& \left.+(F W-D W)^{2}\right], \\
\mathcal{\varepsilon}^{\prime \prime}(\omega)= & (D W+F V)\left(W^{2}+V^{2}\right) / \omega C_{0}\left[(D W+F V)^{2}\right. \\
& \left.+(F W-D V)^{2}\right],
\end{aligned}
$$

где

$$
\begin{gathered}
D=A G M-B G N-B M H-A N H, \\
F=A G N+A M H+B G M-B H N, \\
W=M G-N H+A G-B H+A M-B N, \\
V=N G+M H+B G+A H+B M+A N,
\end{gathered}
$$

где, в свою очередь,

$$
\begin{gathered}
A=R_{\mathrm{I}} /\left(\omega^{2} R_{\mathrm{I}}^{2} C_{\mathrm{I}}^{2}+1\right), \\
B=-\left[\omega^{2} R_{\mathrm{I}}^{2} C_{\mathrm{I}}\left(C_{\mathrm{I}}+C_{b}\right)+1\right] /\left(\omega^{3} R_{\mathrm{I}}^{2} C_{\mathrm{I}}^{2} C_{b}+\omega C_{b}\right), \\
M=R_{\mathrm{II}} /\left(\omega^{2} R_{\mathrm{II}}^{2} C_{\mathrm{II}}^{2}+1\right), \\
N=-\left[\omega^{2} R_{\mathrm{II}}^{2} C_{\mathrm{II}}\left(C_{\mathrm{II}}+C_{m}\right)+1\right] /\left(\omega^{3} R_{\mathrm{II}}^{2} C_{\mathrm{II}}^{2} C_{m}+\omega C_{m}\right) .
\end{gathered}
$$

Выражение (3) позволяет численными методами получить два максимума частотной зависимости $\operatorname{tg} \delta(f)$ (рис. 2), что может служить подтверждением правомочности применения предложенной эквивалентной схемы для анализа экспериментальных результатов.

С помощью выражений (4), (5) получена диаграмма Коула-Коула, т. е. функция $\varepsilon^{\prime \prime}\left(\varepsilon^{\prime}\right)$, построение осуществлялось в параметрическом виде, причем роль параметра играет частота $\omega$. На рис. 2 указанная диаграмма Коула-Коула представлена в виде кривой, содержащей две полуокружности. Получение выражений (3)-(5) символическим методом - второй этап нашего алгоритма.

Следующим, третьим, этапом алгоритма обработки результатов эксперимента являлся анализ выражений (3)-(5) с целью установления возможных реперных точек для однозначного получения численных значений величин $C_{\mathrm{I}}, C_{b}, R_{\mathrm{I}}, C_{\mathrm{II}}, C_{m}, R_{\mathrm{II}}$. Действительно, полученные нами аналитические выражения для $\varepsilon^{\prime}(\omega)$ и $\varepsilon^{\prime \prime}(\omega)$ позволяют найти два корня функции $\varepsilon^{\prime \prime}\left(\varepsilon^{\prime}\right)$, которая в графическом виде представляет собой диаграмму Коула-Коула: $\varepsilon_{0 \mathrm{I}}=\left(C_{b}+C_{m}\right) / C_{0}$ при $\omega \rightarrow 0$ (что соответствует правой абсциссе точки $\varepsilon^{\prime \prime}=0$ на диаграмме Коула-Коула) и $\varepsilon_{0 \text { II }}=$ $=\left(C_{\mathrm{I}} C_{b}\right) /\left[\left(C_{\mathrm{I}}+C_{b}\right) C_{0}\right]+\left(C_{m} C_{\mathrm{II}}\right) /\left[\left(C_{m}+C_{\mathrm{II}}\right) C_{0}\right] \quad$ при $\omega \rightarrow \infty$ (что соответствует левой абсциссе точки $\varepsilon^{\prime \prime}=0$ на диаграмме). Еще две реперные точки являются значениями „высот“ полуокружностей диаграммы Коула-Коула: $h_{\mathrm{I}}=\left\{\left[C_{\mathrm{I}} C_{b} /\left(C_{\mathrm{I}}+C_{b}\right)\right]-C_{b}\right\} / 2 C_{0}, \quad h_{\mathrm{II}}=$ $=\left\{\left[C_{m} C_{\mathrm{II}} /\left(C_{m}+C_{\mathrm{II}}\right)\right]-C_{m}\right\} / 2 C_{0}$. Следующие две peперные точки: $\omega_{0 \mathrm{I}}=(2)^{1 / 2} /\left(R_{\mathrm{I}} C_{\mathrm{I}}\right), \omega_{0 \mathrm{II}}=(2)^{1 / 2} /\left(R_{\mathrm{II}} C_{\mathrm{II}}\right)-$ 
частоты, соответствующие максимумам $\operatorname{tg} \delta(\omega)$. Полученные реперные точки дают систему из 5 уравнений. При этом остается один свободный параметр.

Последним этапом алгоритма является варьирование одного свободного параметра с целью получения наилучшего согласия результатов расчета частотных зависимостей $\operatorname{tg} \delta(f), \varepsilon^{\prime}(f), \varepsilon^{\prime \prime}(f)$ и вида диаграммы Коула-Коула $\varepsilon^{\prime}\left(\varepsilon^{\prime \prime}\right)$ с экспериментально измеряемыми зависимостями. На рис. 2 сплошными линиями показаны результаты такого расчета. Найденное таким образом решение обратной задачи позволяет однозначно определить величины $C_{\mathrm{I}}, C_{b}, R_{\mathrm{I}}, C_{\mathrm{II}}, C_{m}, R_{\mathrm{II}}$, они приведены в подписи к рис. 2.

Обратим внимание на то, что спектрометр марки „Alpha-Beta Impedance Analyzer“ обладает специальными встроенными пакетами программ, позволяющими не только измерять, но и теоретически вычислять частотные зависимости $\operatorname{tg} \delta(f)$ и строить диаграммы Коула-Коула в рамках различных электрических эквивалентных схем исследуемых образцов, в том числе и двухконтурных схем, использованных нами. Однако заранее спрогнозировать численные значения всех 6 параметров для установления однозначного соответствия элементов схемы эксперименту невозможно. Также без анализа выражений (3) -(5) нельзя получить полный вид требуемой системы уравнений, вытекающий из выбора реперных точек. Поэтому использованный нами алгоритм анализа представляется необходимым и оправданным.

Таким образом, можно сделать следующие предварительные выводы.

- Результаты расчета хорошо согласуются с экспериментальными данными, полученными при комнатной температуре (см. рис. 2). Использование реперных точек позволяет выполнить однозначное определение параметров рассмотренных схем.

- Вид экспериментальных диаграмм Коула-Коула, как показывают опыты (см. рис. 3), слабо зависит от температуры.

- Оба максимума частотной зависимости $\operatorname{tg} \delta(\omega, T)$ смещаются с ростом температуры в высокочастотную область (см. рис. 2).

Из сказанного следует, что представляет интерес выполнение более подробного сравнительного исследования температурной зависимости частотного положения двух максимумов функции $\operatorname{tg} \delta(\omega, T)$ и соответствующих им полуокружностей на диаграмме Коула-Коула (здесь $\omega=2 \pi f)$.

\section{5. Температурные зависимости частотного положения максимумов функции $\operatorname{tg} \delta(f)$}

Для нелегированной пленки $\mathrm{VO}_{2}$ на вставке к рис. 2 представлены петли температурного гистерезиса проводимостей $1 / R_{\mathrm{I}}$ и $1 / R_{\text {II }}$ зерен пленки, параметры ветвей которых определяются прямой пропорциональной зависимостью между сопротивлением данной совокупности зерен и частотным положением максимумов: $\left(1 / R_{\mathrm{I}}\right) \propto \omega_{0 \mathrm{I}}$ и $\left(1 / R_{\mathrm{II}}\right) \propto \omega_{0 \text { II. }}$ Обратим внимание на то, что совокупность зерен, обозначенных нами I, имеет электрическое сопротивление $R_{\mathrm{I}}=56$ кОм и в этих зернах происходит структурный фазовый переход Пайерлса при стандартной температуре $T_{c \mathrm{I}}=340 \mathrm{~K}$, тогда как совокупность зерен II имеет электрическое сопротивление $R_{\mathrm{II}}=2$ ГОм и в этих зернах происходит структурный фазовый переход Пайерлса при существенно пониженной температуре, $T_{c \text { II }}=320 \mathrm{~K}$. Это позволяет нам говорить о нормальном и аномальном поведении совокупностей зерен типов I и II соответственно.

Отметим также, что хотя параметры диаграммы Коула-Коула и слабо зависят от температуры, однако рис. 3 показывает все же некоторое уменьшение при росте температуры радиуса низкочастотной окружности, обусловленной совокупностью зерен II типа. Такое уменьшение радиуса в рамках принятой эквивалентной схемы связано с увеличением емкости $C_{\mathrm{II}}$.

\section{6. Обсуждение результатов}

Из того факта, что функция $\operatorname{tg} \delta(f)$ имеет два максимума, $\left(f_{\text {оII }}=1 \Gamma_{ц} \quad\right.$ и $\left.f_{0 \mathrm{I}}=0.5 \mathrm{M} \amalg\right)$, а диаграмма Коула-Коула - две полуокружности (низкочастотную и высокочастотную), следует: в пленке $\mathrm{VO}_{2}$ содержатся два типа релаксаторов (II и I) с постоянными времени $\sim 1 \mathrm{~s}$ и $10^{-5} \mathrm{~s}$. Эти релаксаторы соответствуют двум совокупностям зерен с резко различными электрофизическими свойствами. Действительно, температурные зависимости частотного положения максимумов $\operatorname{tg} \delta(f)$ позволяют получить петли их термического гистерезиса и определить критические температуры мотт-пайерлсовского ФППМ [7] для обеих совокупностей зерен. Оказалось, что низкочастотный максимум имеет $T_{c \text { II }}=320 \mathrm{~K}$, тогда как высокочастотный имеет $T_{c \mathrm{I}}=340 \mathrm{~K}$. Это означает, что у совокупности зерен II типа корреляционные эффекты $[9,10]$ сужают $E_{g}$ нанокристаллитов $\mathrm{VO}_{2}$ и тем самым понижают $T_{c}$ ФППМ сильнее, чем у совокупности зерен I типа. Оказалось, что ширина петли гистерезиса для низкочастотного максимума $(20 \mathrm{~K})$ почти вдвое больше ширины петли высокочастотного максимума $(11 \mathrm{~K})$. Согласно мартенситной модели комплексного мотт-пайерлсовского ФППМ в $\mathrm{VO}_{2}$ [7], это говорит о наличии в пленке $\mathrm{VO}_{2}$ двух резко различающихся по своему поперечнику совокупностей нанокристаллитов: низкочастотный максимум II функции tg $\delta(f)$ соответствует зернам с „малым“ поперечником (широкая петля гистерезиса), тогда как высокочастотный максимум I соответствует зернам с „большим поперечником“ (узкая петля гистерезиса).

О наличии двух типов зерен также говорит наличие ступеньки на нагревной ветви петли температурного гистерезиса электропроводности пленки (см. разд.3), в соответствии с моделью формирования главной петли термического гистерезиса пленки [7]. 
Сравнение экспериментальных данных с расчетными выражениями, полученными для предложенной в данной работе эквивалентной электрической схемы образца пленки $\mathrm{VO}_{2}$, позволяет определить значения всех 6 параметров предложенной схемы (см. подписи к рисункам).

Численные значения $C_{\text {II }}$ и $R_{\text {II }}$ представляют собой электрическую емкость и сопротивление в целом всей совокупности „мелких“ зерен пленки, обеспечивающей электрический суммарный отклик образца пленки на низкой частоте $f_{0 \mathrm{II}} \approx\left(2^{1 / 2}\right) / R_{\mathrm{II}} C_{\mathrm{II}}$. То же имеет место и для полученных в работе численных значениях $C_{\mathrm{I}}$ и $R_{\mathrm{I}}$, соответствующих совокупности „крупных“ зерен пленки на высокой частоте $f_{0 \mathrm{I}} \approx\left(2^{1 / 2}\right) / R_{\mathrm{I}} C_{\mathrm{I}}$.

Обратим внимание на следующее принципиально важное обстоятельство: пониженная температура $T_{c \text { II }}$ ФППМ в зернах с малым поперечником говорит о многократно ( 5 порядков) более высокой общей проводимости, т.е. о низком электрическом сопротивлении, каждого зерна II группы зерен по сравнению с сопротивлением отдельного зерна I группы зерен. С другой стороны, отношение частот $f_{0 \mathrm{I}} / f_{\text {0II }}$ составляет 5 порядков, тогда как электроемкости высокочастотной и низкочастотной совокупностей зерен одного порядка величины $\left(C_{\mathrm{I}}=33\right.$ пФ и $C_{\mathrm{II}}=127$ пФ). Отсюда следует, что различие в 5 порядков времен релаксации, $\tau=(1 / R C)$, обусловлено в первую очередь очень высоким зарядным сопротивлением совокупности зерен II группы $\left(R_{\mathrm{II}} / R_{\mathrm{I}}=10^{5}\right)$ по сравнению с зарядным сопротивлением совокупности зерен I группы. Налицо кажущееся противоречие. Оно может быть снято на основе следующих соображений.

Частотное положение максимума функции $\operatorname{tg} \delta(f)$ определяется временем зарядки суммарной электроемкости данной совокупности зерен в результате протекания тока через зарядное омическое сопротивление. При планарном расположении рабочих электродов в ячейке для регистрации диэлектрических спектров пленки $\mathrm{VO}_{2}$ „крупные“ нанокристаллиты заведомо имеют преимущество в отношении проводимости контакта с электродами ячейки по сравнению с „мелкими“, т.е. сопротивление „электрод-пленка“ $R_{\mathrm{I}}$ для них многократно ниже. Электроды представляют собой полированные латунные диски, покрытые золотой пленкой. Отсюда следует, что „мелкие“ зерна, заряжаясь через гораздо более высокое сопротивление $R_{\mathrm{II}}$, автоматически имеют многократно большее время релаксации и тем самым более низкочастотное положение максимума $\operatorname{tg} \delta(f)$, чем „крупные“.

Подтверждением сказанного служат атомно-силовые изображения поверхности пленки $\mathrm{VO}_{2}$ (рис. 1) и электрода с золотым покрытием. Эти изображения показывают, что шероховатость поверхности золотого покрытия (20 нм) по крайней мере на порядок меньше шероховатости исследованной в настоящей работе пленки $\mathrm{VO}_{2}$ (200-300 нм). Поэтому зарядное сопротивление „мелких “ъ зерен $R_{\mathrm{II}}$ много больше зарядового сопротивления „крупных“ зерен $R_{\mathrm{I}}$.
Итак, положение петли термического гистерезиса и соответственно температура $T_{c}$ ФППМ определяются не сопротивлением контакта, через который осуществляется зарядка соответствующей емкости, а электрическим сопротивлением отдельного $\mathrm{VO}_{2}$-нанокристаллита, т.е. шириной его запрещенной зоны. „Мелкие“ $\mathrm{VO}_{2}$-нанокристаллиты по сравнению с крупными имеют пониженную (320 против $340 \mathrm{~K}$ ) температуру $T_{c}$ ФППМ, уменьшенную ширину запрещенной зоны и, следовательно, меньшее сопротивление отдельного зерна (более высокую проводимость), но большее значение зарядного сопротивления $\left(R_{\mathrm{II}} \gg R_{\mathrm{I}}\right)$. Причина такого положения дел кроется в особенности технологии синтеза пленки $\mathrm{VO}_{2}$, исследованной в настоящей работе.

Как указано в разделе, посвященном экспериментальной методике, нами была использована взрывная методика синтеза пленки $\mathrm{VO}_{2}$. При этом является принципиально важным то, что в данной методике после сбрасывания порции порошка $\mathrm{VO}_{2}$ на разогретую до $770^{\circ} \mathrm{C}$ платформу нанокристаллиты $\mathrm{VO}_{2}$ движутся в вакууме до соприкосновения с подложкой, на которой формируется пленка $\mathrm{VO}_{2}$. В процессе такого движения нагретые нанокристаллиты теряют через свою поверхность уходящий в вакуум кислород, создавая кислородные вакансии, обладающие донорными свойствами [11]. Нанокристаллиты с малым поперечником приобретают более высокую концентрацию кислородных вакансий (и, тем самым, доноров) ввиду относительно большего вклада поверхностных слоев в общий объем нанокристаллита, нежели „крупные“. Таким образом, „мелкие“ нанокристаллиты, имея более высокую концентрацию доноров, имеют более узкую благодаря корреляционным эффектам запрещенную зону, чем „крупные“ [7], и отсюда более низкую температуру $T_{c}$ пайерлсовской части ФППМ.

Подчеркнем, что предложенный в настоящей работе алгоритм сопоставления экспериментальных результатов с результатами математического расчета параметров предлагаемой эквивалентной схемы позволяет получать численное значение отношения площадей подложки $S$, занятых упомянутыми совокупностями „мелких“ и „крупных“ зерен. Это отношение равно 1.2. Оно обеспечивается возможностью раздельного определения из диаграммы Коула-Коула емкостей подложки $C_{m}=7.5$ пФ и $C_{b}=6.2$ пФ, которые пропорциональны этим площадям.

Таким образом, особенности механизма ФППМ в кристаллических пленках $\mathrm{VO}_{2}$ заключаются в следующем. По мере увеличения температуры структурный фазовый переход при критической температуре $T_{c \text { II }}$ вначале совершают „мелкие“ зерна, несмотря на их относительно широкую элементарную петлю гистерезиса. Это опережение связано с тем, что температура равновесия фаз $T_{0 I I}$ „мелких“ зерен, соответствующая середине элементарной петли, ниже $T_{0 I}$,крупных“ зерен. Вслед за фазовым переходом в „мелких“ зернах происходит структурный фазовый переход также и в „крупных“ зернах при $T_{c \mathrm{I}}$. В результате различия критических темпе-

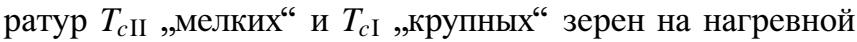


ветви главной петли температурного гистерезиса пленки формируется ступенька.

Итак, можно констатировать, что описанные особенности механизма ФППМ в пленках $\mathrm{VO}_{2}$ обусловлены разделением зерен пленки при ее синтезе на две совокупности: „крупных“ и „мелких“ зерен. „Мелкие“ зерна имеют бо́льшую концентрацию свободных электронов из-за возникающих в процессе синтеза пленки вакансий кислорода. Вследствие этого благодаря корреляционным эффектам они имеют меньшую ширину запрещенной зоны и, следовательно, меньшее значение $T_{0 \text { II. Идея о }}$ роли зерен разных размеров в механизме ФППМ в пленках $\mathrm{VO}_{2}$ высказывалась неоднократно [7]. Однако в настоящей работе методами ДС удалось впервые экспериментально разделить вклады „крупных“ и „мелких“ зерен в ФППМ и показать, что эти вклады действительно разнесены по величинам критических температур. Оказалось, что метод ДС имеет то преимущество, что позволяет раздельно идентифицировать электрофизические параметры различных совокупностей нанокристаллитов, перемешанных на поверхности пленки $\mathrm{VO}_{2}$ в случайном порядке.

\section{Конфликт интересов}

Авторы заявляют, что у них нет конфликта интересов.

\section{Список литературы}

[1] A.V. Ilinskiy, O.E. Kvashenkina, E.B. Shadrin. Smart Nanocomposites, 4 (2), 65 (2014).

[2] A. Velichko, M. Belyaev, V.V. Putrolainen. Intern. J. Mod. Phys., 31 (2), 1234 (2016).

[3] А.С. Олейник. Автореф. докт. дис. (Саратов, 2008).

[4] О.Б. Данилов, В.А. Климов, О.П. Михеева, А.И. Сидоров, С.А. Тульский, Е.Б. Шадрин, И.Л. Ячнев. ЖТФ, 73 (1), 79 (2003).

[5] F. Kremer, A. Schonhals, W. Luck. Broadband Dielectric Spectroscopy (Springer Verlag, 2002).

[6] Ю.А. Зейгарник, Ю.П. Ивочкин, В.С. Григорьев, А.А. Оксман. ТВТ, 46 (5), 797 (2008).

[7] Е.Б. Шадрин, А.В. Ильинский, А.И. Сидоров, С.Д. Ханин. ФТT, 52 (11), 2269 (2010)

[8] А.В. Ильинский, Р.А. Кастро, А.А. Кононов, М.Э. Пашкевич, Е.Б. Шадрин. ЖТФ, 45 (11), 877 (2019).

[9] А.В. Ильинский, О.Е. Квашенкина, Е.Б. Шадрин. ФТП, 46 (4), 439 (2012).

[10] А.В. Ильинский, М.Э. Пашкевич, Е.Б. Шадрин. НТВ СПбГПУ, ФМН, 10 (3), 9 (2017).

[11] П. Кофстад. Отклонение от стехиометрии, дифбузия и электропроводность в простых окислах металлов (М., Мир, 1975).

Редактор Л.В. Шаронова

\section{Dielectric spectroscopy and features of the semiconductor-metal phase transition mechanism in $\mathbf{V O}_{2}$ films}

\author{
A.V. llinskiy ${ }^{1}$, R.A. Kastro ${ }^{2}$, M.E. Pashkevich ${ }^{3}$, \\ E.B. Shadrin ${ }^{1}$ \\ ${ }^{1}$ loffe Institute, \\ 194021 St. Petersburg, Russia \\ ${ }^{2}$ Herzen State Pedagogical University, \\ 191186 St. Petersburg, Russia \\ ${ }^{3}$ Peter the Great St.Petersburg Polytechnic University, \\ 195251 St. Petersburg, Russia
}

Abstract In the range $0.1-10^{6} \mathrm{~Hz}$, the temperature transformation of the frequency dependences of the dielectric loss tangent $\tan [\delta(f)]$, as well as the Cole-Cole diagrams for undoped vanadium dioxide films, were investigated. The measurements were carried out in the temperature range $T=273-373 \mathrm{~K}$. It has been shown that the form of the Cole-Cole diagrams for all films weakly depended on the temperature in the indicated interval, while the frequencies $f_{0}$ corresponding to the maxima of the function $\tan [\delta(f)]$ increased with temperature. The temperature hysteresis loops of the frequency positions of the maxima $f_{0}(T)$ were measured. In interpreting the data of dielectric spectroscopy, a complex equivalent electrical circuit of the sample was used, which made it possible to detect the presence of two types of grains with different electrical properties in undoped $\mathrm{VO}_{2}$ films. The presence of two types of grains determines the features of the semiconductor-metal phase transition mechanism in $\mathrm{VO}_{2}$ films. 